\title{
Too little radiation pressure on dust in the winds of oxygen-rich AGB stars
}

\begin{abstract}
P. Woitke
Leiden University, PO Box 9513, 2300 RA Leiden, The Netherlands

e-mail: woitke@strw.leidenuniv.nl

Received 30 August 2006 / Accepted 6 October 2006

ABSTRACT

Aims. It is commonly assumed that the massive winds of AGB stars are dust-driven and pulsation-enhanced. However, detailed frequency-dependent dynamical models that can explain the observed magnitudes of mass loss rates and outflow velocities have been published so far only for C-stars. This letter reports on first results of similar models for oxygen-rich AGB stars. The aim is to provide a better understanding of the wind driving mechanism, the dust condensation sequence, and the role of pulsations.

Methods. New dynamical models for dust-driven winds of oxygen-rich AGB stars are presented which include frequency-dependent Monte Carlo radiative transfer by means of a sparse opacity distribution technique and a time-dependent treatment of the nucleation, growth and evaporation of inhomogeneous dust grains composed of a mixture of $\mathrm{Mg}_{2} \mathrm{SiO}_{4}, \mathrm{SiO}_{2}, \mathrm{Al}_{2} \mathrm{O}_{3}, \mathrm{TiO}_{2}$, and solid Fe.

Results. The frequency-dependent treatment of radiative transfer reveals that the gas is cold close to the star $\left(700-900 \mathrm{~K}\right.$ at $\left.1.5-2 R_{\star}\right)$ which facilitates the nucleation process. The dust temperatures are strongly material-dependent, with differences as large as $1000 \mathrm{~K}$ for different pure materials, which has an important influence on the dust formation sequence. Two dust layers are formed in the dynamical models: almost pure glassy $\mathrm{Al}_{2} \mathrm{O}_{3}$ close to the $\operatorname{star}\left(r \gtrsim 1.5 R_{\star}\right)$ and the more opaque Fe-poor $\mathrm{Mg}$-Fe-silicates further out. Solid Fe and Fe-rich silicates are found to be the only condensates that can efficiently absorb the stellar light in the near IR. Consequently, they play a key role in the wind driving mechanism and act as a thermostat. Only small amounts of Fe can be incorporated into the grains, because otherwise the grains become too hot. Thus, the models reveal almost no mass loss, and no dust shells.

Conclusions. The observed dust sequence $\mathrm{Al}_{2} \mathrm{O}_{3} \rightarrow \mathrm{Fe}$-poor $\mathrm{Mg}$-Fe-silicates for oxygen-rich AGB stars having low $\rightarrow$ high mass loss rates is in agreement with the presented model and can be understood as follows: $\mathrm{Al}_{2} \mathrm{O}_{3}$ is present in the extended atmosphere of the star below the wind acceleration region, also without mass loss. The Mg-Fe-silicates form further out and, therefore, their amount depends on the mass loss rate. The driving mechanism of oxygen-rich AGB stars is still an unsolved puzzle.
\end{abstract}

Key words. hydrodynamics - radiative transfer - stars: winds, outflows - stars: mass-loss - stars: AGB and post-AGB

\section{Introduction}

The mass loss mechanism of AGB stars and red supergiants is a long-standing astrophysical problem. In the carbon-rich case, an extraordinary condensate exists (amorphous carbon) which is very stable, i.e. it can exist close the star, and is very opaque in the optical and near IR spectral region. Detailed dynamical models with time-dependent dust formation (Winters et al. 2000) show that the formation of amorphous carbon can provide sufficient radiation pressure to drive massive outflows, consistent with the basic characteristics of C-star winds. This result has been confirmed by dynamical models with frequency-dependent radiative transfer by Höfner et al. (2003).

However, in the oxygen-rich case, no such condensate exists. The most stable metal oxides like $\mathrm{Al}_{2} \mathrm{O}_{3}$ are too rare. The abundant pure silicates like $\mathrm{Mg}_{2} \mathrm{SiO}_{4}$ are less stable and almost completely transparent around $1 \mu \mathrm{m}$ where most of the stellar flux escapes. Solid Fe and $\mathrm{Mg}$-Fe-silicates are opaque but even less stable. Stationary models of dust-driven O-rich AGB star winds with grey radiative transfer (Ferrarotti \& Gail 2006) and dynamical models with pulsation and grey radiative transfer (Jeong et al. 2003) nevertheless suggest that the winds of O-rich AGB stars are dust-driven, where the stellar pulsation helps to provide the necessary density conditions to form the dust close to the star ("pulsation-enhanced").
In contrast, the a posteriori frequency-dependent radiative transfer analysis of non-linear pulsation models with simplified dust formation theory by Ireland \& Scholz (2006) did not find much radiation pressure on dust $\left(\mathrm{Al}_{2} \mathrm{O}_{3}\right.$ and $\left.\mathrm{Mg}_{2 x} \mathrm{Fe}_{2-2 x} \mathrm{SiO}_{4}\right)$ in O-rich Mira variables, with radiative accelerations as small as 0.08 to 0.29 times the gravitational deceleration.

Recent mid-IR observations of O-rich AGB stars in globular clusters with SPITZER (Lebzelter et al. 2006) and of galactic bulge AGB stars with ISO (Blommaert et al. 2006) show a clear correlation between the kind of condensate and the mass loss rate $\dot{M}$, called the "observational dust condensation sequence": stars with low $\dot{M}$ show mainly $\mathrm{Al}_{2} \mathrm{O}_{3}$, whereas stars with higher $\dot{M}$ show increasing amounts of $\mathrm{Mg}$-Fe-silicates. From MIDI interferometry of the red supergiant $\alpha$ Ori, Verhoelst et al. (2006) concluded that $\mathrm{Al}_{2} \mathrm{O}_{3}$ grains are already present at radial distances as small as $r=1.5 R_{\star}$.

\section{The model}

Hydrodynamics is solved by using the FLASH-solver (Fryxell et al. 2000) in spherical symmetry, including gravity and selfdeveloped modules for radiation pressure on dust \& molecules and radiative heating/cooling (see Woitke 2006a for details). In this letter, we use the piston approximation as inner boundary condition to simulate the pulsation of the star, and a new 


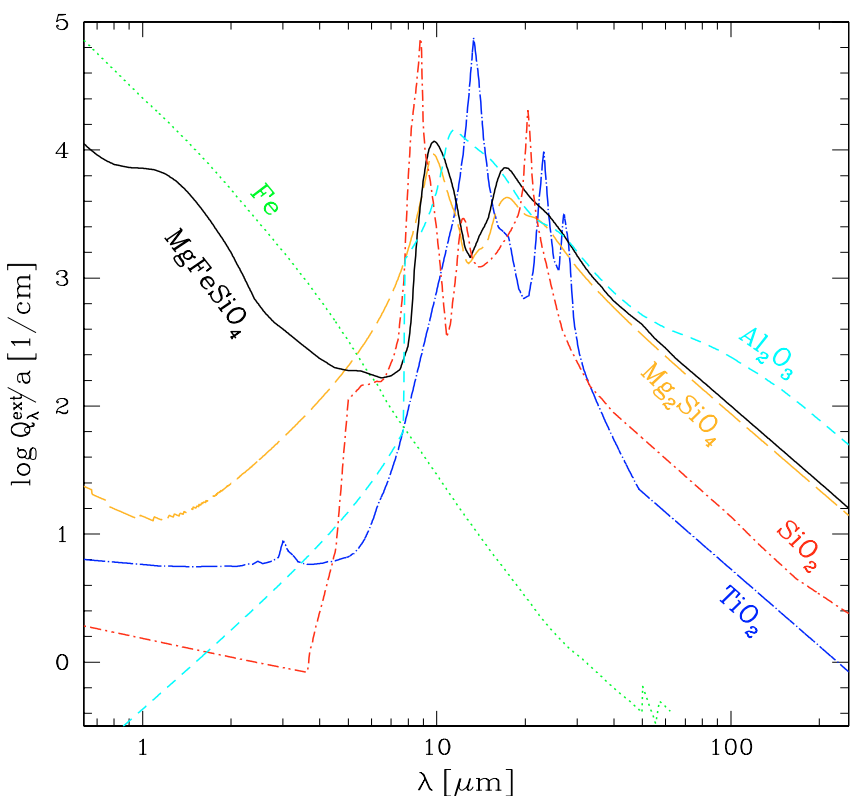

Fig. 1. Extinction efficiencies over particle radius $Q_{\text {ext }} / a$ in the Rayleigh-limit of Mie theory according to the Jena optical data base. The data is partly $\log -\log$ extrapolated.

equation of state for a mixture of $\mathrm{H}^{+}, \mathrm{e}^{-}, \mathrm{H}, \mathrm{H}_{2}, \mathrm{He}$ and other atomic metals in LTE, including ionisation and dissociation potentials, and vibrational and rotational excitation energies of $\mathrm{H}_{2}$.

Radiative transfer: For the models presented in this letter, we have developed a new frequency-dependent Monte Carlo radiative transfer technique which allows for arbitrarily high optical depths (Woitke 2006b). In frequency-dependent stellar atmospheres, the gas is always optically thick at least in some wavelengths $\left(\sim 10^{5}\right)$, which is a significant problem for standard MC techniques. The method is coupled to the hydrodynamics and can be used also for 2D models.

Opacities: The basis for our radiative transfer treatment are monochromatic molecular gas opacities from the MARCS stellar atmosphere code (Jørgensen et al. 1992), extracted by Helling et al. (2000). The frequency space is subdivided into five spectral bands with two opacity distribution points in each band, resulting in altogether $5 \times 2$ effective wavelength sampling points (for the traditional ODF approximation see e.g. Carbon 1979). High and low mean opacity values are tabulated for each spectral band during the initialisation phase of the program in such a way that they simultaneously result in the correct Planck and Rosseland band-mean gas opacities. The details will be explained in another paper (Woitke 2006b).

Dust opacities are calculated in the Rayleigh limit of Mie theory according to the Jena optical data base, kindly provided by Th. Posch (see Fig. 1). The total dust extinction coefficient $\left[\mathrm{cm}^{2} / \mathrm{g}\right]$ is assumed to be given by ${ }^{1}$

$\hat{\kappa}_{\lambda, \mathrm{ext}}^{\mathrm{dust}}=\frac{3}{4} L_{3} \sum_{s} \frac{V_{s}}{V_{\mathrm{tot}}} Q_{\mathrm{ext}}^{s}(a, \lambda) / a$

where $L_{3}$ is the third moment of the dust size distribution function and $V_{s} / V_{\text {tot }}$ is the volume fraction of solid material $s$ in

\footnotetext{
${ }^{1}$ According to our assumption of inhomogeneous grains, an application of the effective medium theory would be more appropriate, which will be examined in a future paper. Preliminary results show that the effective extinction is stronger than the simple volume-means used in this paper.
}

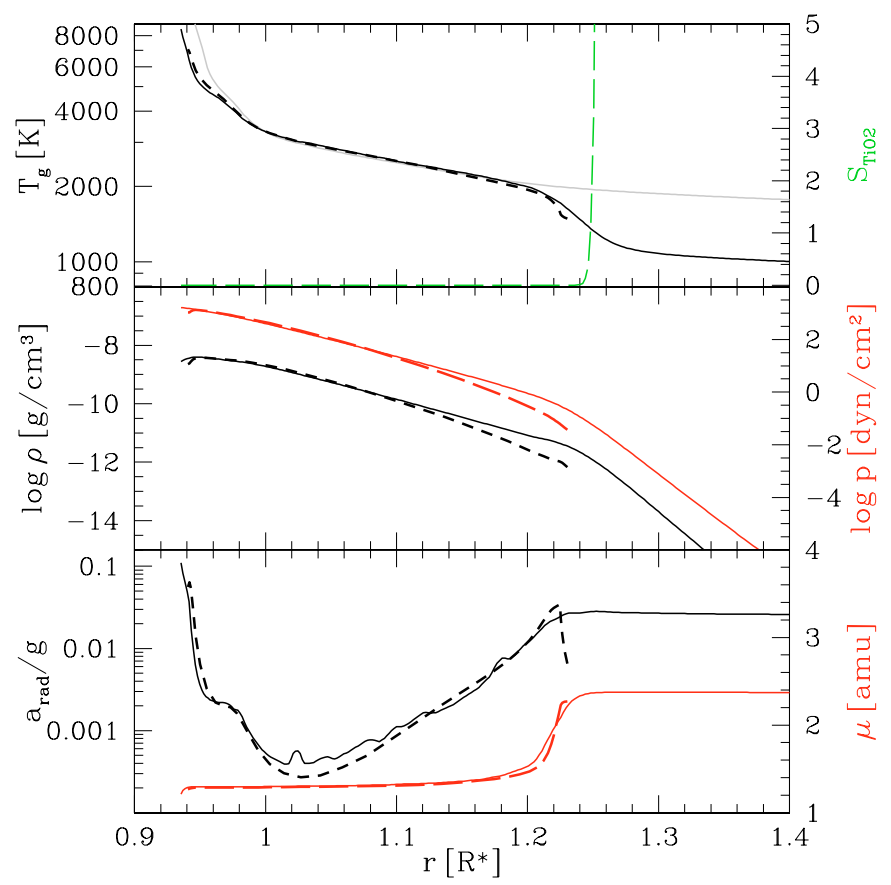

Fig. 2. Hydrostatic initial model (full) in comparison to a spherical MARCS model (dashed) and a grey model (grey $T_{\mathrm{g}}$-line) for $M_{\star}=1 M_{\odot}$, $T_{\star}=2800 \mathrm{~K}, \log g=-0.6\left(L_{\star}=6048 L_{\odot}\right), Z=1 . S_{\mathrm{TiO} 2}$ shows the supersaturation ratio of $\mathrm{TiO}_{2}$, indicating that nucleation is already possible very close to the star. Long-dashed graphs belong to the r.h.s. axis.

the dust component. The extinction efficiencies over particle radius of the pure solids $s$ are shown in Fig. 1. Note that most oxygen-rich condensates have a "glassy" character. They are almost transparent in the optical and near IR but opaque in the mid IR where the strong vibrational resonances are situated. In contrast, solid Fe and Fe-rich silicates are opaque even in the optical. The monochromatic dust opacities are subject to the same averaging procedure to result in high/low band-mean dust opacities as described above for the gas opacities.

Dust formation is described by a system of differential moment equation explained in Helling \& Woitke (2006) considering the growth and evaporation of inhomogeneous dust grains composed of a mixture of $\mathrm{Mg}_{2} \mathrm{SiO}_{4}, \mathrm{SiO}_{2}, \mathrm{Al}_{2} \mathrm{O}_{3}, \mathrm{TiO}_{2}$, and solid $\mathrm{Fe}$ (13 growth/evaporation reactions). The nucleation rate of $\left(\mathrm{TiO}_{2}\right)_{\mathrm{N}}$ clusters is adopted from Jeong (2000). The molecular concentrations entering into the calculation of the nucleation and growth rates are calculated by a small neutral equilibrium chemistry for 11 atoms $(\mathrm{H}, \mathrm{He}, \mathrm{C}, \mathrm{O}, \mathrm{N}, \mathrm{Mg}, \mathrm{Al}, \mathrm{Si}, \mathrm{S}, \mathrm{Ti}, \mathrm{Fe})$ and 33 molecules $\left(\mathrm{H} 2, \mathrm{CO}, \mathrm{CO}_{2}, \mathrm{OH}, \mathrm{H}_{2} \mathrm{O}, \mathrm{CH}_{4}, \mathrm{~N}_{2}, \mathrm{CN}, \mathrm{HCN}\right.$, $\mathrm{NH}_{3}, \mathrm{H}_{2} \mathrm{~S}, \mathrm{SiS}, \mathrm{SO}, \mathrm{HS}, \mathrm{SiO}, \mathrm{SiH}, \mathrm{SiH}_{4}, \mathrm{SiO}_{2}, \mathrm{SiN}, \mathrm{SO}_{2}, \mathrm{MgH}$, $\mathrm{MgS}, \mathrm{MgO}, \mathrm{MgOH}, \mathrm{Mg}(\mathrm{OH})_{2}, \mathrm{FeO}, \mathrm{Fe}(\mathrm{OH})_{2}, \mathrm{AlOH}, \mathrm{AlO}_{2} \mathrm{H}$, $\left.\mathrm{Al}_{2} \mathrm{O}, \mathrm{AlH}, \mathrm{TiO}, \mathrm{TiO}_{2}\right)$.

\section{The static solution}

The hydrostatic, dust-free solution (see Woitke 2006a for equations and further details) used as initial model for the dynamical models is shown in Fig. 2. The obtained degree of agreement with the MARCS model is remarkable for such a rough 10-wavelength-point treatment of radiative transfer. Similar results have been obtained by Höfner et al. (2003) and Helling \& Jørgensen (1998), using a sparse opacity sampling technique with about 100 sampling points. The gas temperature structure $T_{\mathrm{g}}(r)$ shows a pronounced "step" of almost $1000 \mathrm{~K}$ 
Table 1. Calculated dust temperatures $T_{\mathrm{d}}$ (first row) and dust radiative accelerations $\Gamma_{\text {dust }}=a_{\text {ras }}^{\text {dust }} / g$ (second row) in the case of full condensation into small particles in the static model (see Fig. 2). The resulting dust-to-gas ratio $\rho_{\text {dust }} / \rho_{\text {gas }}$ is shown in the middle column. Temperatures values with ${ }^{\star}$ mark thermally unstable condensates.

\begin{tabular}{|c|c|c|c|c|}
\hline solid material & $\frac{\rho_{\text {dust }}}{\rho_{\text {gas }}}\left[10^{-3}\right]$ & $r=1.5 R_{\star}$ & $r=2 R_{\star}$ & $r=5 R_{\star}$ \\
\hline \multirow[t]{2}{*}{$\mathrm{TiO}_{2}$} & 0.0061 & $1030 \mathrm{~K}$ & $750 \mathrm{~K}$ & $380 \mathrm{~K}$ \\
\hline & & 0.00004 & 0.00004 & 0.00005 \\
\hline \multirow[t]{2}{*}{$\mathrm{Al}_{2} \mathrm{O}_{3}$} & 0.11 & $1090 \mathrm{~K}$ & $810 \mathrm{~K}$ & $420 \mathrm{~K}$ \\
\hline & & 0.0013 & 0.0014 & 0.0015 \\
\hline \multirow[t]{2}{*}{$\mathrm{SiO}_{2}$} & 1.6 & $1000 \mathrm{~K}$ & $740 \mathrm{~K}$ & $380 \mathrm{~K}$ \\
\hline & & 0.032 & 0.034 & 0.036 \\
\hline \multirow[t]{2}{*}{$\mathrm{Mg}_{2} \mathrm{SiO}_{4}$} & 1.9 & $1150 \mathrm{~K}$ & $850 \mathrm{~K}$ & $430 \mathrm{~K}$ \\
\hline & & 0.022 & 0.024 & 0.025 \\
\hline \multirow[t]{2}{*}{$\mathrm{MgFeSiO}_{4}$} & 4.0 & $1930 \mathrm{~K}^{\star}$ & $1710 \mathrm{~K}^{\star}$ & $1170 \mathrm{~K}$ \\
\hline & & 1.3 & 1.4 & 1.4 \\
\hline \multirow[t]{2}{*}{$\mathrm{MgSiO}_{3}$} & 2.3 & $1010 \mathrm{~K}$ & $740 \mathrm{~K}$ & $380 \mathrm{~K}$ \\
\hline & & 0.025 & 0.027 & 0.029 \\
\hline \multirow[t]{2}{*}{$\mathrm{Mg}_{0.5} \mathrm{Fe}_{0.5} \mathrm{SiO}_{3}$} & 3.0 & $1880 \mathrm{~K}^{\star}$ & $1580 \mathrm{~K}^{\star}$ & $690 \mathrm{~K}$ \\
\hline & & 0.21 & 0.21 & 0.18 \\
\hline \multirow[t]{2}{*}{$\mathrm{Fe}$} & 1.3 & $1980 \mathrm{~K}^{\star}$ & $1770 \mathrm{~K}^{\star}$ & $1280 \mathrm{~K}$ \\
\hline & & 0.85 & 0.89 & 0.88 \\
\hline am. carbon & 3.0 & $1870 \mathrm{~K}^{\star}$ & $1640 \mathrm{~K}$ & $1130 \mathrm{~K}$ \\
\hline$(\mathrm{C} / \mathrm{O}=1.5)$ & & 20 & 21 & 21 \\
\hline
\end{tabular}

around $1.25 R_{\star}$ (the MARCS model is not extended enough to reveal this step completely). This is not just a "surface effect". Outside of this $T_{\mathrm{g}}$-step even the strongest molecular lines become optically thin (in the hydrostatic case) and the line blanketing effect works at full strength. The grey model fails completely in predicting this step which is very meaningful for the dust formation. The agreement with the MARCS results concerning the mass density $\rho(r)$, the gas pressure $p(r)$, the mean molecular weight $\mu(r)$, and the acceleration by radiation pressure on molecules divided by gravity $\Gamma_{\text {gas }}=a_{\text {rad }} / g(r)$ is also good.

\section{Rough estimates of the dust acceleration}

The calculation of the spectral mean intensities $J_{\lambda}(r)$ and spectral fluxes $F_{\lambda}(r)$ in the static model (see Fig. 2) allows for a quick estimate of the maximum possible radiative acceleration by dust if the dust is still optically thin. Table 1 shows the resulting dust temperatures $T_{\mathrm{d}}$ of several pure condensates

$\int \hat{\kappa}_{\lambda, \text { abs }}^{\text {dust }} J_{\lambda}(r) \mathrm{d} \lambda=\int \hat{\kappa}_{\lambda, \text { abs }}^{\text {dust }} B_{\lambda}\left(T_{\mathrm{d}}\right) \mathrm{d} \lambda$

and the radiative acceleration by dust divided by gravity

$\Gamma_{\text {dust }}(r)=\frac{\frac{1}{c} \int \hat{\kappa}_{\lambda, \text { ext }}^{\text {dust }} F_{\lambda}(r) \mathrm{d} \lambda}{\frac{G M(r)}{r^{2}}}$

at three selected distances from the star. For each condensate we take the maximum possible dust volume per mass $L_{3}$, max given by element conservation constraints, e.g. $L_{3, \max }^{\mathrm{Mg}_{2} \mathrm{SiO}_{4}}=$ $\operatorname{Min}\left\{\frac{1}{2} \epsilon_{\mathrm{Mg}}, \epsilon_{\mathrm{Si}}, \frac{1}{4} \epsilon_{\mathrm{O}}\right\} V_{\mathrm{Mg}_{2} \mathrm{SiO}_{4}} n_{\langle\mathrm{H}\rangle} / \rho$ where $n_{\langle\mathrm{H}\rangle}$ is the hydrogen nuclei density, $\epsilon_{k}$ the abundance of element $k$ and $V_{\mathrm{Mg}_{2} \mathrm{SiO}_{4}}$ the monomer volume of $\mathrm{Mg}_{2} \mathrm{SiO}_{4}$ (see Helling \& Woitke 2006).

The results shown in Table 1 demonstrate that the dust temperatures $T_{\mathrm{d}}$ are strongly material-dependent, with differences as large as $1000 \mathrm{~K}$ at the same distance from the star, which is a remarkable result. All condensates (except solid Fe) have strongly peaked mid-IR resonances which are situated just around the maximum of the local Planck function - they work perfectly for radiative cooling. In contrast, the glassy character of the oxides and pure silicates like $\mathrm{Al}_{2} \mathrm{O}_{3}, \mathrm{SiO}_{2}, \mathrm{Mg}_{2} \mathrm{SiO}_{4}$ and $\mathrm{MgSiO}_{3}$ (the low absorption efficiencies at optical and near-IR wavelengths, see Fig. 1) prevent efficient heating by the star. Consequently, the pure glassy condensates can exist astonishingly close to the star (see also Woitke 1999).

For the same reasons, radiative pressure on all glassy condensates is negligible! It is hence without effect for the wind acceleration mechanism whether for example $\mathrm{Mg}_{2} \mathrm{SiO}_{4}$ condenses out or not. The only dust species that can potentially drive a stellar outflow are solid $\mathrm{Fe}$ and $\mathrm{Fe}$-rich silicates like $\mathrm{MgFeSiO}_{4}$.

The unavoidable consequence of the spectral characteristics of oxygen-rich dust is that radiative acceleration must be paid for by radiative heating, i.e. dust species capable of driving a stellar wind $(\Gamma \gtrsim 1)$ are hot and can only exist at a relatively large distance from the star (e.g. $r \gtrsim 5 R_{\star}$, marked in bold in Table 1). In comparison, amorphous carbon is so opaque and stable (in a C-rich gas) that it could accelerate the gas already outwards of $2 R_{\star}$, with $20 \times$ the local gravity in this model.

\section{Results of the dynamical models}

The first results of the dynamical models showed almost no mass loss $\left(\dot{M} \lesssim 10^{-10} M_{\odot} / \mathrm{yr}\right)$, just some erratic large-scale and long-term excursions for which the mass loss rate is difficult to measure. We then approached rather extreme stellar parameters $\left(M_{\star}=1 M_{\odot}, T_{\star}=2500 \mathrm{~K}, L_{\star}=10000 L_{\odot}\right)$, still without success. Finally, in order to see what a dust-driven wind could look like, we arbitrarily enhanced the radiative acceleration by

$\Gamma=\Gamma_{\text {gas }}+5 \Gamma_{\text {dust }}$.

The results of this simulation is shown in Fig. 3.

The models (also those without enhanced $\Gamma$ ) show extended warm molecular layers, truncated by the $T_{\mathrm{g}}$-step described in Sect. 3 due to the line blanketing effect. The pulsation of the star leads to a time-dependent extension of these layers to roughly $1.5-2 R_{\star}$. If dust forms, it fills in the gas opacity gaps in frequency space which reduces the line blanketing effect.

The formation of seed particles (see the nucleation rate $J_{\star} / n_{\langle\mathrm{H}\rangle}$ in Fig. 3) happens right above these molecular layers, i.e. very close to the star. The gas is cold here $\left(T_{\mathrm{g}} \approx 700-900 \mathrm{~K}\right)$ which is not revealed by models using grey radiative transfer (Jeong et al. 2003; Ferrarotti \& Gail 2006).

According to the model, two dust layers develop: almost pure glassy $\mathrm{Al}_{2} \mathrm{O}_{3}$ grains close to the $\operatorname{star}\left(r \gtrsim 1.5 R_{\star}\right.$, partly inside the warm molecular layers where $T_{\mathrm{g}} \approx T_{\mathrm{d}} \lessgtr 1500 \mathrm{~K}$ ), and further out the more opaque $\mathrm{Mg}$-Fe-silicates which grow on top of the $\mathrm{Al}_{2} \mathrm{O}_{3}$ particles with a very small, steadily increasing Fe content.

The temperature of the inhomogeneous dust grains is controlled by the iron content, which has already been noted by Tielens et al. (1998). The volume fraction of solid iron inclusions relaxes quickly to a level where a further increase would cause too much radiative heating and thus thermal re-evaporation of the solid iron inclusions

$T_{\mathrm{d}} \approx T_{\mathrm{S}}^{\mathrm{Fe}}(\rho)$

where $T_{\mathrm{S}}^{\mathrm{Fe}}$ is the sublimation temperature of solid iron. This is a robust and recurrent result concerning many simulations, because there is a stable self-regulation mechanism: the iron content adapts quickly to any changes in the ambient medium (density, radiation field) unless the density becomes too small out in the wind (here at $r \gtrsim 5 R_{\star}$ ). Consequently, the degree of condensation of Fe only reaches a final level of $\sim 17 \%$. It is this 


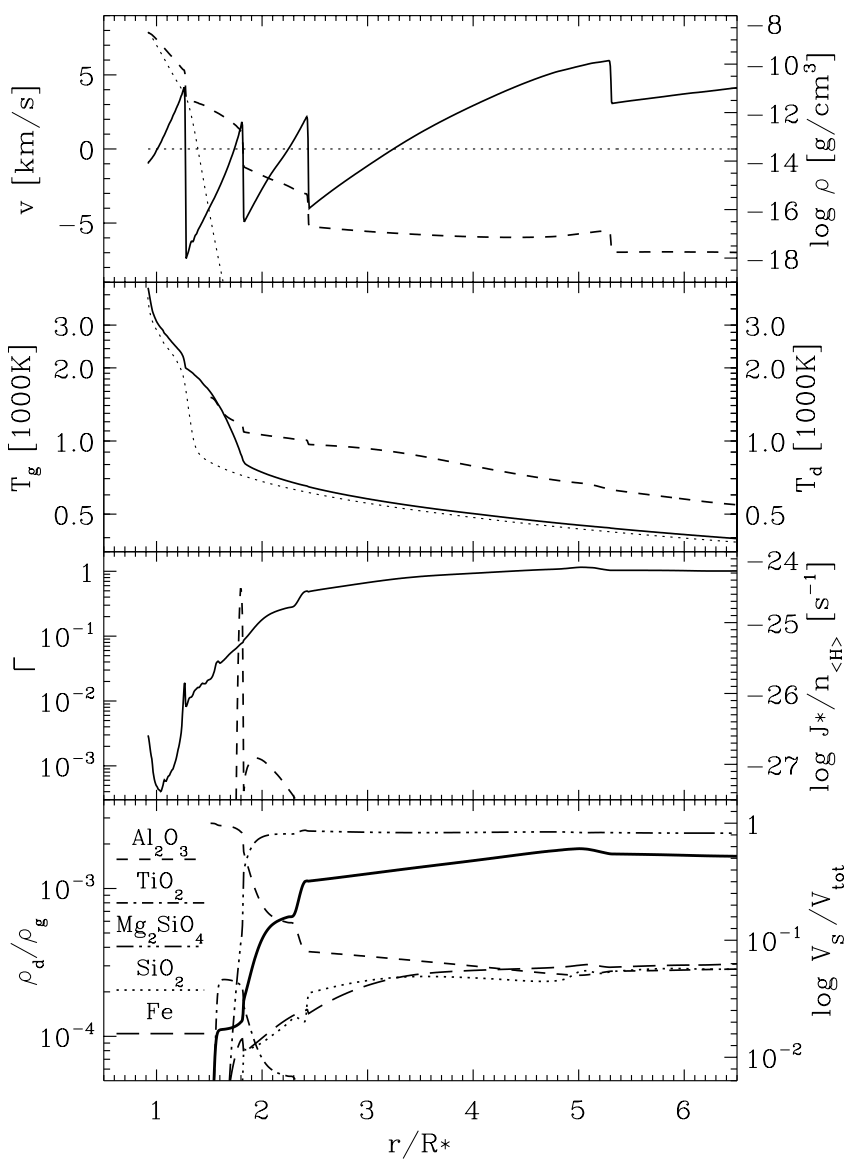

Fig. 3. Dynamical wind model after 100 years of simulation time. Parameter: $M_{\star}=1 M_{\odot}, T_{\star}=2500 \mathrm{~K}, L_{\star}=10000 L_{\odot}(\log g=$ $-1.015), Z=1$, piston period $P=600$ days and velocity amplitude $\Delta v=2 \mathrm{~km} \mathrm{~s}^{-1}$. The dotted curves show the hydrostatic solution. The dashed graphs belong to the r.h.s. axis. $\Gamma$ is arbitrarily enhanced (see text).

low value that kills the mass loss (in comparison $\mathrm{Mg}: \sim 65 \%, \mathrm{Al}$ : $\sim 100 \%$ ). Interestingly, silicates in AGB star winds are in fact observed to be Fe-poor (Bowey et al. 2002).

The radiative acceleration just exceeds the gravitational deceleration around $r \approx 3.5-4 R_{\star}$, which can be considered as an analog of the sonic point in stationary winds. Determined by long-term averages at the outer boundary, the mean mass loss rate, the mean outflow velocity and the mean dust-to-gas ratio are $\langle\dot{M}\rangle \approx 2.3 \times 10^{-9} M_{\odot} / \mathrm{yr},\left\langle v_{\infty}\right\rangle \approx 2.6 \mathrm{~km} \mathrm{~s}^{-1}$ and $\left\langle\rho_{\mathrm{d}} / \rho_{\mathrm{g}}\right\rangle \approx$ $1.6 \times 10^{-3}$, respectively (for $\Gamma=\Gamma_{\text {gas }}+5 \Gamma_{\text {dust }}$ ).

\section{Conclusions}

1. This letter reports on a negative result. Detailed dynamical models with frequency-dependent Monte Carlo radiative transfer and time-dependent formation of inhomogeneous dust grains cannot explain the observed magnitude of mass loss rates from oxygen-rich AGB stars, even in case of extreme stellar parameters (i.e. high $L_{\star} / M_{\star}$ ratios).

2. The role of solid iron and Fe-rich silicates is crucial for the wind driving mechanism. These condensates are the only ones that are opaque around $1 \mu \mathrm{m}$ and, thus, only these condensates can efficiently absorb the stellar light. Since the Fe containing condensates are not particularly stable, they form at too large distances from the star in order to provide an efficient mass loss mechanism.
3. Previous grey models (Jeong et al. 2003; Ferrarotti \& Gail 2006) have calculated the radiation pressure on dust with Rosseland mean opacities which leads to a severe overestimation in the O-rich case. There is a mismatch between the maximum of the stellar flux around $1 \mu \mathrm{m}$ and the strongly peaked dust opacities around $10-20 \mu \mathrm{m}$, which is not well described by the grey approximation. The integral in Eq. (3) is smaller, because the local flux is not $B_{\lambda}\left(T_{\mathrm{d}}\right)$-like.

4. The dust condensation sequence is strongly affected by radiative transfer effects. Pure, glassy condensates like $\mathrm{Al}_{2} \mathrm{O}_{3}$ have lower dust temperatures than solid $\mathrm{Fe}$ or $\mathrm{Fe}$-rich silicates. The differences are as large as $1000 \mathrm{~K}$, which favours the formation of the glassy condensates and prevents the formation of $\mathrm{Fe}$-inclusions close to the star. The results in this paper are consistent with the observational finding of $\mathrm{Al}_{2} \mathrm{O}_{3}$ at radial distances as small as $1.5 R_{\star}$ around $\alpha$ Ori (Verhoelst et al. 2006) as well as with the observed dust condensation sequence in O-rich AGB stars (Blommaert et al. 2006; Lebzelter et al. 2006), because $\mathrm{Al}_{2} \mathrm{O}_{3}$ can exist in an extended atmosphere without mass loss, whereas the $\mathrm{Mg}$-Fe-silicates form in the more distant wind regions which require mass loss.

5. The mass loss mechanism of oxygen-rich AGB stars and red supergiants is still a puzzle. Pulsations alone cannot drive an outflow because the radiative cooling of the gas is too efficient, even in non-LTE (Woitke 2003; Schirrmacher et al. 2003). According to the results of this letter, even a combination of stellar pulsation and radiation pressure on dust is insufficient to drive the mass loss. Do we have to re-visit Alfvén-waves (e.g. Vidotto \& Jatenco-Pereira 2006)?

Acknowledgements. This work is part of the ASTROHYDRO3D initiative supported by the NWO COMPUTATIONAL PHYSICS PROGRAMME, grant 614.031.017. The computations have been done on the parallel Xeon cluster LISA in Almere, the Netherlands, SARA grant MP-103. The software used in this work was in part developed by the DOE-supported ASCI/Alliance Center for Astrophysical Thermonuclear Flashes at the University of Chicago.

\section{References}

Blommaert, J. A. D. L., Groenewegen, M. A. T., Okumura, K., et al. 2006, A\&A, 460, 555

Bowey, J. E., Barlow, M. J., Molster, F. J., et al. 2002, MNRAS, 331, L1

Carbon, D. F. 1979, ARA\&A, 17, 513

Ferrarotti, A. S., \& Gail, H.-P. 2006, A\&A, 447, 553

Fryxell, B., Olson, K., Ricker, P., Timmes, F. X., Zingale, M., et al. 2000, ApJ, 131, 273

Helling, Ch., \& Jørgensen, U. G. 1998, A\&A, 337, 477

Helling, Ch., Winters, J. M., \& Sedlmayr, E. 2000, A\&A, 358, 651

Helling, Ch., \& Woitke, P. 2006, A\&A, 455, 325

Höfner, S., Gautschy-Loidl, R., Aringer, B., \& Jørgensen, U. G. 2003, A\&A, 399, 589

Ireland, M. J., \& Scholz, M. 2006, MNRAS, 367, 1585

Jeong, K. S. 2000, Ph.D. Thesis, Technische Universität, Berlin, Germany

Jeong, K. S., Winters, J. M., Le Bertre, T., \& Sedlmayr, E. 2003, A\&A, 407, 191

Jørgensen, U. G., Johnson, H. R., \& Nordlund, А 1992, A\&A, 261, 263

Lebzelter, T., Posch, T., Hinkle, K., Wood, P. R., \& Bouwman, J. 2006, ApJL, submitted

Schirrmacher, V., Woitke, P., \& Sedlmayr, E. 2003, A\&A, 404, 267

Tielens, A. G. G. M., Waters, L. B. F. M., Molster, F. J., \& Justanont, K. A\&SS, 255, 415

Verhoelst, T., Decin, L., van Malderen, R., et al. 2006, A\&A, 447, 311

Vidotto, A. A., \& Jatenco-Pereira, V. 2006, ApJ, 639, 416

Winters, J. M., Le Bertre, T., Jeong, K. S., Helling, Ch., \& Sedlmayr, E. 2000, A\&A, 361, 641

Woitke, P. 1999, in Astronomy with Radioactivities, ed. R. Diehl, \& D. Hartmann (Schloß Ringberg, Germany) MPE Rep. 274, 163

Woitke, P. 2006a, A\&A, 452, 537

Woitke, P. 2006b, MNRAS, in prep.

Woitke, P. 2003, IAU Symp., 210, 387 